\title{
Search as Learning (SAL) Workshop 2016
}

\author{
Jacek Gwizdka \\ University of Texas Austin, USA \\ sigir2016@gwizdka.com
}

\author{
Preben Hansen \\ Stockholm University, Sweden \\ preben@dsv.su.se
}

\author{
Claudia Hauff \\ Delft University of Technology, \\ the Netherlands \\ c.hauff@tudelft.nl
}

\author{
Jiyin $\mathrm{He}$ \\ Centrum Wiskunde \& Informatica, \\ the Netherlands \\ jiyinhe@acm.org
}

\author{
Noriko Kando \\ National Institute of \\ Informatics, Japan \\ kando@nii.ac.jp
}

\begin{abstract}
The "Search as Learning" (SAL) workshop is focused on an area within the information retrieval field that is only beginning to emerge: supporting users in their learning whilst interacting with information content.
\end{abstract}

\section{Keywords}

Search, learning, human information interaction

\section{MOTIVATION}

Search systems to date are viewed more as tools for the retrieval of content to satisfy immediate information needs, than as part of larger complex information environments in which humans learn while interacting with information content. As users increasingly learn informally while searching as well as use search systems as tools for self-study, there is a growing recognition of the importance to address the challenges of designing, developing, and evaluating search systems that foster discovery and enhance learning outside of formal educational settings.

The research agenda of "Search as Learning" aims to bring together these challenges and opportunities by reaching out to researchers with backgrounds in information science (IS), human computer interaction (HCI), and information retrieval (IR), with the goal of integrating conceptual, experimental, and simulation-based approaches and methodologies from within these different fields. This will allow the transformation of search systems as isolated information access tools into systems that provide support for learning directly and that consider the broader outcomes of searching beyond a set of search results.

Studies in IS have always focused on the broader context of search with the aim to understand, conceptualise, and form theories of the relations between user behavior and the users' information environment. Earlier work identified search as not to be an isolated activity but part of a larger information seeking process, i.e., "a process, in which humans purposefully engage in order to change their state of knowledge" [12]. Further work proposed that the information seeking processes should be thought of as driven

Permission to make digital or hard copies of part or all of this work for personal or classroom use is granted without fee provided that copies are not made or distributed for profit or commercial advantage and that copies bear this notice and the full citation on the first page. Copyrights for third-party components of this work must be honored. For all other uses, contact the owner/author(s).

SIGIR '16 July 17-21, 2016, Pisa, Italy

(C) 2016 Copyright held by the owner/author(s).

ACM ISBN 978-1-4503-4069-4/16/07.

DOI: http://dx.doi.org/10.1145/2911451.2917766 by higher-level human needs or the user environment, e.g, in the context of a work task [1]. The importance of learning has resurfaced as noted by Jansen et al. [10]: "a learning theory may better describe the information searching process than more commonly used paradigms of decision making or problem solving". Connecting learning theory and IS perspectives [13] suggests that "the use of information as the fundamental building block for learning". In the first edition of this workshop (SAL'14) Freund et al. [7] presented a conceptual framework for the role of search in informal learning and Byström [2] discussed the social, individual and techno-material dimensions of search as a learning activity in the context of real-life work tasks and how this leads to future research topics and methodological implications for interactive information retrieval. These conceptual models and frameworks provide theoretical foundations for exploratory analyses of log data as well as the development of empirical models to validate the proposed theories. Recently, Vakkari [17] and Rieh et al. [15] reviewed relevant research so far and discussed future directions from both theoretical and empirical perspective [8].

Within the IR and HCI community studies addressing different aspects of learning during search are gaining traction, while following a more empirical, data-driven methodology, e.g., studies that analyse user learning behaviour from commercial search logs [5, 18], and that investigate behavioural indicators of the evolving status of users' knowledge during search $[4,6,19]$. Further, the need to develop evaluation paradigms that go beyond individual query interaction has been noted by many IR researchers. A number of directions have been proposed, e.g., towards whole session based evaluation, user-centric evaluation methods and metrics $[3,9,14$, 16], as well as evaluation of search outcome in broader contexts such as work tasks [11]. The effort of integrating whole-session and task based evaluation of IR systems with IR's test-collection driven simulation based evaluation paradigm is reflected by the recent editions of the TREC Session Track and Tasks Track. The interactive and context-rich nature of learning as both a process and outcome of information seeking is the next frontier in the evaluation of information systems and is a natural fit for the strong evaluation focussed IR community.

Together, the theory from IS and empirical models from IR studies have the necessary prerequisites to shed light on how local evaluation of ranking and system design within a particular part of the search process can be combined with more global assessments of system-user performance. On the one hand, conceptual models and frameworks of the cognitive process of searching and learning would provide guidance to use the empirical results in appropriate contexts; and on the other hand, empirical predictive models and simulation based evaluation would provide means to translate the 
conceptual models and frameworks into operations at an algorithmic level. However, it is not yet well understood how the results of these different lines of research should be put together and operationalised for the design and evaluation of IR systems that support learning activities.

SIGIR is a key venue that brings together researchers from IS, $\mathrm{HCI}$, and IR, and the SAL workshop provides the opportunity where theory meets empirical studies. The discussion in the workshop will not only identify and prioritise the problems and solutions on the topic of search as learning, but also contribute to the future of IR research in seeking integrated cognitive and empirical approaches to the theory, modelling, and evaluation of information seeking processes and information systems.

\section{THEME AND PURPOSE}

The Search as Learning Workshop aims to flesh out research directions and methodologies and survey state-of-the-art approaches in this important emerging research area. We are particularly interested in engaging researchers across the IR, IS, HCI and learning science fields.

Topics of interest include the following but not limited to:

- Understanding searching as a human learning process;

- Learning process in the context of work tasks;

- System features and functionalities to foster learning;

- The implications of searching for learning for different populations: children, low literacy searchers, non-experts;

- The role of affect and engagement on learning during search;

- Evaluation of learning performance and experience;

- Learning analytics for search contexts;

- Collaborative aspects of searching as learning;

- Interaction monitoring, modelling, and optimization for learning outcomes.

\section{WORKSHOP FORMAT AND PLANNED ACTIVITIES}

The workshop includes two keynotes presentations (Kevyn CollinsThompson from University of Michigan and Andreas Nuernberger from University of Magdeburg), presentations of selected papers, and interactive sessions in the format of breakout groups.

The focus of the workshop is to encourage interaction and collaboration among attendees. With the interactive sessions, we expect to identify and define the major challenges from the perspective of different research areas, and to explore and discover interdisciplinary challenges. Overall, we expect the workshop to foster new research directions for search and learning and future collaborations such as joint projects and publications.

\section{REFERENCES}

[1] P. Borlund. The IIR evaluation model: a framework for evaluation of interactive information retrieval systems. Information research, 8(3), 2003.

[2] K. Byström. Searching as a learning activity in real life workplaces. In Workshop on Search as Learning (SAL) 2014., 2014.

[3] M. Cole, J. Liu, N. Belkin, R. Bierig, J. Gwizdka, C. Liu, J. Zhang, and X. Zhang. Usefulness as the criterion for evaluation of interactive information retrieval. Proc. HCIR, pages 1-4, 2009.
[4] M. J. Cole, J. Gwizdka, C. Liu, N. J. Belkin, and X. Zhang. Inferring user knowledge level from eye movement patterns. Inf. Process. Manage., 49(5):1075-1091, Sept. 2013.

[5] C. Eickhoff, J. Teevan, R. White, and S. Dumais. Lessons from the journey: a query log analysis of within-session learning. In Proceedings of the 7th ACM international conference on Web search and data mining, pages 223-232. ACM, 2014.

[6] C. Eickhoff, S. Dungs, and V. Tran. An eye-tracking study of query reformulation. In Proceedings of the 38th International ACM SIGIR Conference on Research and Development in Information Retrieval, pages 13-22. ACM, 2015.

[7] L. Freund, H. O'Brien, and R. Kopak. Getting the big picture: supporting comprehension and learning in search. In Workshop on Search as Learning (SAL) 2014., 2014.

[8] P. Hansen and S. Y. Rieh. Editorial: Recent advances on searching as learning: An introduction to the special issue. Journal of Information Science, 42(1):3-6, 2016.

[9] J. He, M. Bron, A. de Vries, L. Azzopardi, and M. de Rijke. Untangling result list refinement and ranking quality: a framework for evaluation and prediction. In Proceedings of the 38th International ACM SIGIR Conference on Research and Development in Information Retrieval, pages 293-302. ACM, 2015.

[10] B. J. Jansen, D. Booth, and B. Smith. Using the taxonomy of cognitive learning to model online searching. Information Processing \& Management, 45(6):643-663, 2009.

[11] K. Järvelin and P. Ingwersen. Information seeking research needs extension towards tasks and technology. Information Research, 10(1), 2004.

[12] G. Marchionini. Information seeking in electronic environments. Cambridge university press, 1997.

[13] D. Neuman. Learning in information-rich environments. In Encyclopedia of the Sciences of Learning, pages 1908-1910. Springer, 2012.

[14] K. Raman, P. N. Bennett, and K. Collins-Thompson. Toward whole-session relevance: Exploring intrinsic diversity in web search. In Proceedings of the 36th international ACM SIGIR conference on Research and development in information retrieval, pages 463-472. ACM, 2013.

[15] S. Y. Rieh, K. Collins-Thompson, P. Hansen, and H.-J. Lee. Towards searching as a learning process: A review of current perspectives and future directions. Journal of Information Science, 42(1):19-34, 2016.

[16] M. D. Smucker and C. L. Clarke. Time-based calibration of effectiveness measures. In Proceedings of the 35th international ACM SIGIR conference on Research and development in information retrieval, pages 95-104. ACM, 2012.

[17] P. Vakkari. Searching as learning: A systematization based on literature. Journal of Information Science, 42(1):7-18, 2016.

[18] R. W. White, S. T. Dumais, and J. Teevan. Characterizing the influence of domain expertise on web search behavior. In Proceedings of the Second ACM International Conference on Web Search and Data Mining, pages 132-141. ACM, 2009.

[19] X. Zhang, M. Cole, and N. Belkin. Predicting users' domain knowledge from search behaviors. In Proceedings of the 34th International ACM SIGIR Conference on Research and Development in Information Retrieval, pages 1225-1226. ACM, 2011. 